\title{
FGF21 is an endocrine signal of protein restriction
}

\author{
Thomas Laeger, ${ }^{1}$ Tara M. Henagan, ${ }^{1}$ Diana C. Albarado, ${ }^{1}$ Leanne M. Redman, ${ }^{1}$ George A. Bray, ${ }^{1}$ Robert C. Noland, ${ }^{1}$ \\ Heike Münzberg, ${ }^{1}$ Susan M. Hutson, ${ }^{2}$ Thomas W. Gettys, ${ }^{1}$ Michael W. Schwartz, ${ }^{3}$ and Christopher D. Morrison ${ }^{1}$ \\ 1Pennington Biomedical Research Center (PBRC), Baton Rouge, Louisiana, USA. ${ }^{2}$ Virginia Polytechnic Institute and State University, Blacksburg, Virginia, USA. ${ }^{3}$ Diabetes and Obesity Center of Excellence, \\ Department of Medicine, University of Washington, Seattle, Washington, USA
}

\begin{abstract}
Enhanced fibroblast growth factor 21 (FGF21) production and circulation has been linked to the metabolic adaptation to starvation. Here, we demonstrated that hepatic FGF21 expression is induced by dietary protein restriction, but not energy restriction. Circulating FGF21 was increased 10-fold in mice and rats fed a low-protein (LP) diet. In these animals, liver Fgf21 expression was increased within 24 hours of reduced protein intake. In humans, circulating FGF21 levels increased dramatically following 28 days on a LP diet. LP-induced increases in FGF21 were associated with increased phosphorylation of eukaryotic initiation factor $2 \alpha$ (elF2 $\alpha$ ) in the liver, and both baseline and LP-induced serum FGF21 levels were reduced in mice lacking the elF2 $\alpha$ kinase general control nonderepressible 2 (GCN2). Finally, while protein restriction altered food intake, energy expenditure, and body weight gain in WT mice, FGF21-deficient animals did not exhibit these changes in response to a LP diet. These and other data demonstrate that reduced protein intake underlies the increase in circulating FGF21 in response to starvation and a ketogenic diet and that FGF21 is required for behavioral and metabolic responses to protein restriction. FCF21 therefore represents an endocrine signal of protein restriction, which acts to coordinate metabolism and growth during periods of reduced protein intake.
\end{abstract}

\section{Introduction}

Nutrient restriction engages a variety of metabolic and behavioral adaptations, and in the case of energy restriction, these adaptations are well described as including alterations in feeding behavior, energy expenditure (EE), and insulin sensitivity. Dietary protein intake is also critical for survival, and while several lines of evidence support the hypothesis that protein intake is regulated (1-3), little is known regarding how the body detects dietary protein restriction or engages adaptive metabolic and behavioral responses (4).

Fibroblast growth factor 21 (FGF21) is a humoral signal implicated in the adaptive response to both starvation and ketogenic diets (KDs) (5-8), and interventions that raise circulating FGF21 levels increase insulin sensitivity and EE, among other effects (9-14). Although FGF21 is expressed in multiple tissues, increases in its circulating levels have been linked to increased FGF21 synthesis in the liver, while the metabolic and behavioral effects of this hormone involve actions in brain, liver, and adipose tissue $(13,15-20)$. Despite these data, some uncertainty remains regarding the physiological role of FGF21 (21). Plasma levels of FGF21 are paradoxically increased in the settings of obesity and insulin resistance (22-24), and unlike in rodents, plasma FGF21 levels are not consistently increased by KDs or fasting in humans $(23,25,26)$. These discrepancies highlight the need for a better understanding of the upstream regulatory mechanisms that control FGF21 synthesis and secretion as well as its physiological role in metabolic regulation.

\section{Related Commentary: p. 3691}

Conflict of interest: The authors have declared that no conflict of interest exists Submitted: December 23, 2013; Accepted: June 5, 2014.

Reference information: J Clin Invest. 2014;124(9):3913-3922. doi:10.1172/JCI74915.
Based on similarities between the metabolic response to FGF21 administration and dietary protein restriction, we sought in the current work to determine whether FGF21 plays a physiological role in the response to dietary protein restriction. We report that restricted protein intake, independent of energy intake, reliably increases circulating FGF21 levels and hepatic expression of FGF21 in both mice and rats, and reproduce the effect of protein restriction on plasma FGF21 in humans. We further establish that reduced protein intake underlies the previously reported effects of both starvation and KD to increase circulating FGF21 levels. Consistent with prior work linking hepatic FGF21 to ER stress and the depletion of single essential amino acids (27-30), we also demonstrate that general control nonderepressible 2 (GCN2)/eukaryotic initiation factor $2 \alpha$ (eIF $2 \alpha$ ) signaling is an upstream regulator of FGF21 during protein restriction. Finally, the behavioral and metabolic responses to dietary protein restriction displayed by normal mice are absent in mice lacking FGF21. Taken together, these data suggest that FGF21 is a signal of protein restriction rather than energy restriction and offer an explanation for the effect of dietary protein deficiency on behavior and metabolism.

\section{Results}

FGF21 is rapidly and robustly induced by dietary protein restriction in rodents and humans. Rats consuming low-protein (LP) diet exhibited increased food intake (control: $23.53 \pm 0.9 \mathrm{~g} / \mathrm{d}$; LP: $28.06 \pm$ $1.2 \mathrm{~g} / \mathrm{d}, P=0.01)$, consistent with previous work $(31,32)$. Although there was no effect of diet on final body weight or body weight gain, 14 days of LP diet increased body adiposity (control: $18.2 \%$ $\pm 0.005 \%$; LP: $20.3 \% \pm 0.008 \%$ fat, $P=0.034$ ). Consistent with a state of dietary protein restriction (33), LP diet altered circulating amino acid concentrations, reduced hepatic leucine oxidation, 
A

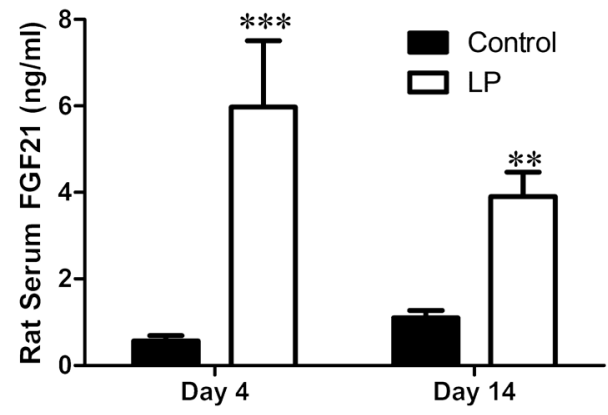

B

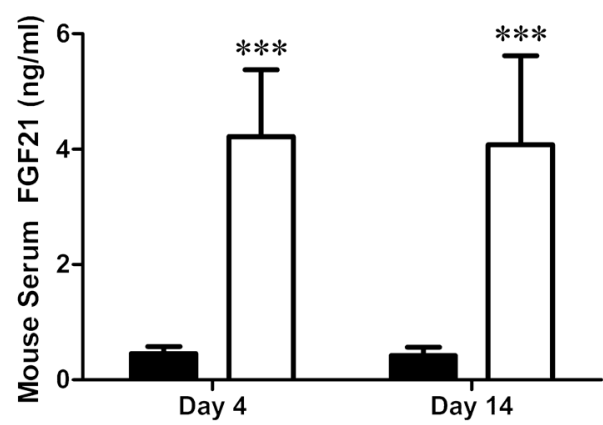

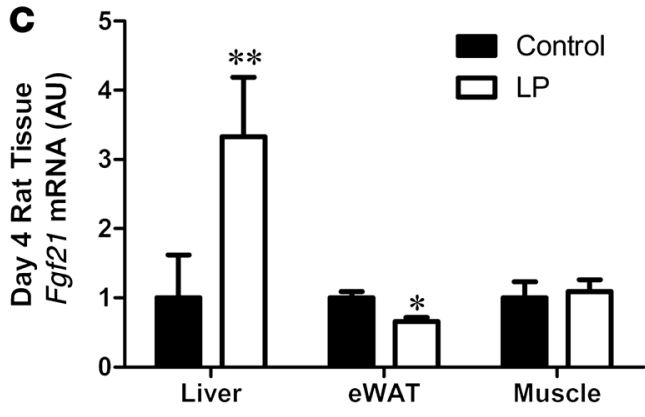

D

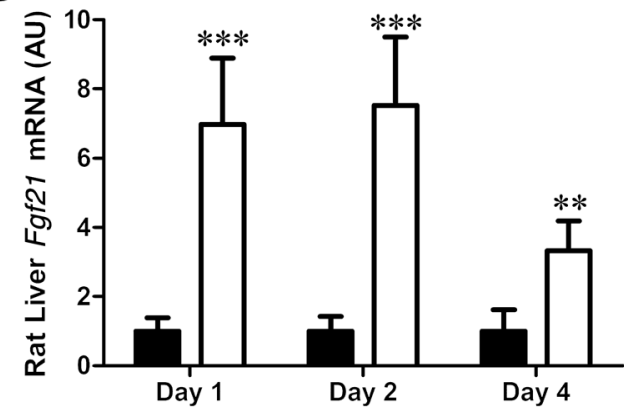

Figure 1. FGF21 is increased by dietary protein restriction in rodents. Rats (A) and mice (B) were placed on isocaloric control or LP diets for 4 or 14 days, with circulating FGF21 protein levels assessed by ELISA. Fgf21 mRNA expression was measured in rat liver, eWAT, and muscle (C) following 4 days of LP. Acute changes in liver Fgf21 mRNA expression in rats were measured over time (D). $n=5$-8/group; ${ }^{*} P<0.05 ;{ }^{* *} P<0.01 ;{ }^{* *} P<0.001$ vs. control.

and increased the expression of genes involved in hepatic amino acid biosynthesis (3-phosphoglycerate dehydrogenase [3PGD] and asparagine synthetase [ASNS], Supplemental Figure 1; supplemental material available online with this article; doi:10.1172/ JCI74915DS1). Consumption of the LP diet markedly increased serum FGF21 concentrations in both rats and mice after either 4 or 14 days $(P<0.001$, Figure $1, A$ and $B)$. To investigate the source of the increased FGF21, we measured Fgf21 mRNA expression in rat liver, adipose tissue, and skeletal muscle after 4 days on the LP diet. While hepatic Fgf 21 mRNA expression was significantly increased at day 4, there was no increase in Fgf21 mRNA expression in either epididymal white adipose tissue (eWAT) or muscle (Figure 1C). Hepatic Fgf21 mRNA expression was increased 7-fold after 24 hours of LP diet $(P<0.001$; Figure 1D). Collectively, these data indicate that dietary protein restriction is a potent stimulator of hepatic and circulating FGF21.

Protein restriction, but not energy restriction, increases circulating FGF21 levels. To test the hypothesis that protein restriction is responsible for the increase in circulating FGF21 levels during restriction/starvation (8), rats were exposed to 4 dietary conditions for 4 days, leading to restriction of either protein alone (LP:normal energy $[\mathrm{NE}]$ ), energy alone (normal protein $[\mathrm{NP}]$ :low energy [LE]), or both protein and energy (LP:LE; Supplemental Figure 2, A-C). Protein-restricted rats exhibited unique changes in hepatic gene expression that were clearly distinguishable from hepatic gene expression of energy restricted rats, as protein restriction but not energy restriction increased the expression of liver 3Pgd and Asns (Supplemental Figure 2, $\mathrm{D}-\mathrm{F}$ ). As observed previously, protein restriction in the absence of energy restriction (LP:NE) robustly increased both circulating FGF21 levels and hepatic Fgf21 mRNA expression $(P<0.01$;
Figure 2, A and B). In contrast, NP but restricted energy intake (NP:LE) decreased circulating FGF21 protein and hepatic Fgf21 mRNA expression, whereas combined protein and energy restriction produced a modest doubling of circulating FGF21 protein and hepatic Fgf21 expression. These data demonstrate that protein restriction powerfully increases hepatic and circulating FGF21, whereas the opposite effect is observed when energy but not protein is restricted.

To examine this finding in greater detail, we assessed the effects of refeeding rats either a LP diet (5\% protein) or a high-protein (HP) diet (40\% protein) in equal amounts following 48 hours of food deprivation. Energy intake (Supplemental Figure 3A) and body weight (Supplemental Figure 3C) during the refeeding period were identical between the 2 refed groups, but protein intake was markedly different, as expected (Supplemental Figure 3B). Whereas starvation increased circulating FGF21 levels $(P=0.06$, Figure 2C), refeeding the LP diet resulted in a 5-fold larger increase in FGF21 than starvation alone $(P<0.01)$. This response was not observed in response to HP refeeding, despite a similar energy intake, indicating that FGF21 is more robustly induced under conditions in which energy intake is maintained but protein intake is reduced, as compared with conditions of total food restriction in which both energy and protein intake are reduced.

$L P$ intake underlies the increase in FGF21 on a KD. We hypothesized that the previously reported increase of plasma FGF21 concentrations in mice fed a $\operatorname{KD}(7,34)$ could potentially derive from protein restriction, since the diet used in these studies is low in protein (4.7\% of energy; Supplemental Table 1). To test this hypothesis, mice were placed on one of 4 diets: (a) control; (b) KD alone; (c) KD supplemented with carbohydrate (KD + $\mathrm{CHO}$ ); or (d) KD supplemented with an equal amount of protein $(\mathrm{KD}+\mathrm{P})$. Consistent with 
A

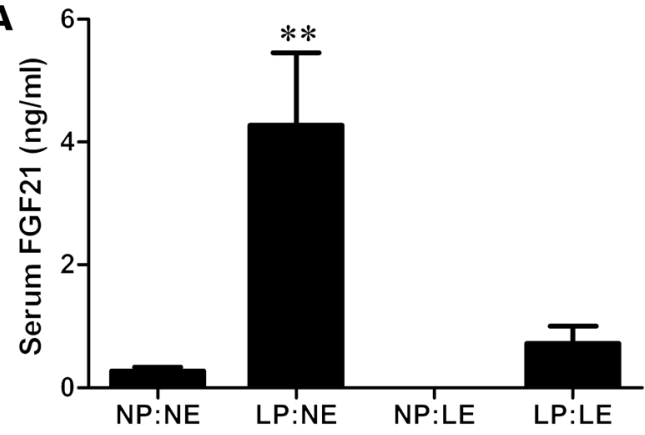

B
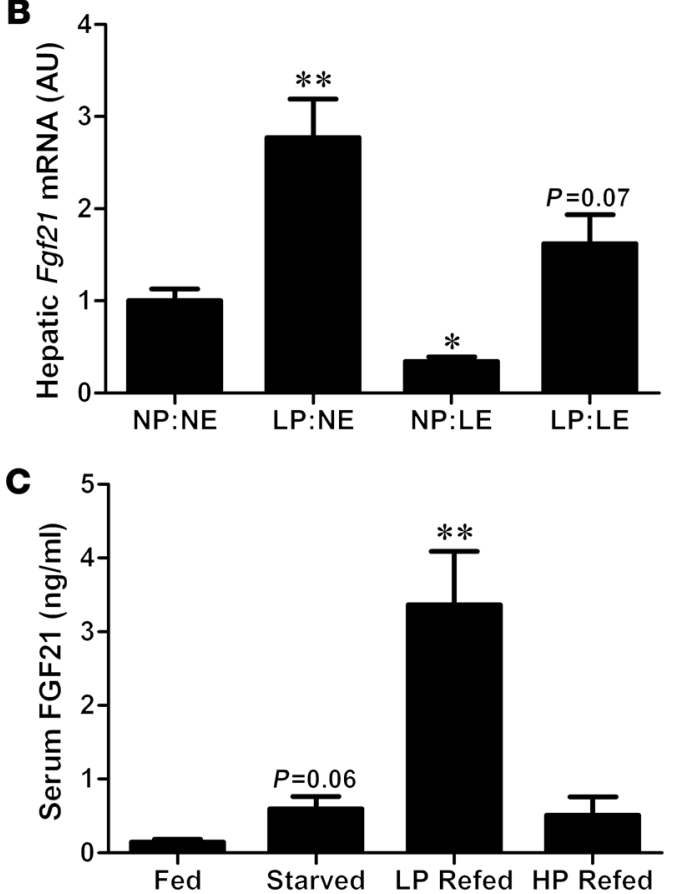
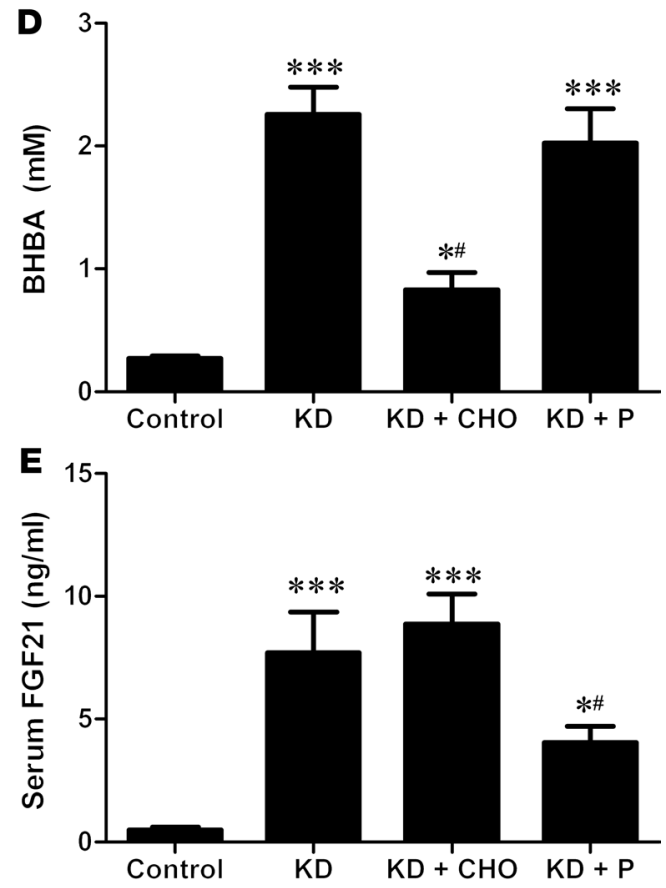

Figure 2. Protein restriction underlies the increase in FGF21 during food restriction or a KD. Serum FGF21 (A) and liver Fgf21 mRNA expression (B) were compared in rats placed on diets that independently restricted energy vs. protein for 4 days: control (NP:NE), LP:NE, NP:LE, and LP:LE. Serum FGF21 was also compared in a separate group of rats (C) that were food deprived for 48 hours and then refed equal caloric amounts of either a LP or HP diet. Serum BHBA (D) and FGF21 (E) were measured in mice fed control, KD, or KD supplemented with an isocaloric amount of either carbohydrate (KD + CHO) or protein (KD + P) for 4 days. $n=5$ /group; ${ }^{*} P<0.05 ;{ }^{* *} P<0.01 ;{ }^{* *} P<0.001$ vs. control/NP:NE; ${ }^{*} P<0.05$ vs. KD.

previous reports, KD feeding for 4 days increased circulating levels of both the ketone body ß-hydroxybutyric acid (BHBA) (Figure 2D; $P<0.001$ ) and FGF21 (Figure 2E; $P<0.001$ ). Supplementation of the $\mathrm{KD}$ with $\mathrm{CHO}$ significantly reduced BHBA relative to $\mathrm{KD}$ $(P<0.05)$, but had no effect on FGF21. In contrast, supplementing the KD with protein had no effect on BHBA, but attenuated the KD-induced increase in FGF21 levels by approximately 50\% (Figure 2E). These data suggest that the increase of FGF21 during KD is primarily driven by the low level of dietary protein, rather than the ketotic state or reduced dietary carbohydrate.

Protein restriction increases FGF21 in humans. We next tested whether dietary protein restriction without energy restriction also increases FGF21 in humans. FGF21 levels were measured in human plasma samples collected as part of a randomized controlled clinical trial (35) in which subjects were overfed $(\sim 0 \%$ above energy needs) diets that varied in protein content. Whereas plasma FGF21 concentrations did not change significantly between baseline and day 28 in subjects fed the control diet $(P=0.40$; Figure $3 \mathrm{~A})$, plasma FGF21 increased between day 0 and day 28 in subjects on the LP diet ( $P=0.06$; Figure $3 B$ ). Expressed as percentage change from baseline, FGF21 concentrations increased by $171 \%$ in LP-fed subjects $(P=0.008$, Figure 3C). These data suggest that, just as in rodents, circulating FGF21 increases in humans in response to dietary protein restriction. Interestingly, 3 control and 2 LP subjects exhibited FGF21 concentrations that were unusually high compared with the remaining subjects in the study. The high FGF21 values were apparent in both the baseline (day 0 ) and day 28 samples and thus are not due to the dietary manipulation. Although the basis for this elevation is currently unclear, prior studies document a high degree of variability in FGF21 concentrations among individuals (26).

$L P$-induced increases in FGF21 depend on both GCN2 and PPAR signaling. The effect of starvation and KD on increasing hepatic Fgf21 gene expression has been attributed to activation of the transcription factor PPAR $\alpha$, a mediator of numerous hepatic responses to nutrient restriction $(7,8)$. However, we observed no increase 
A

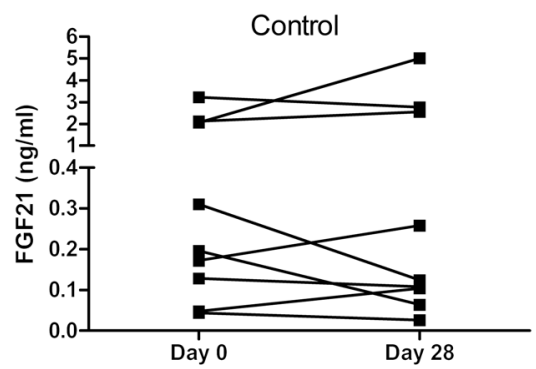

B
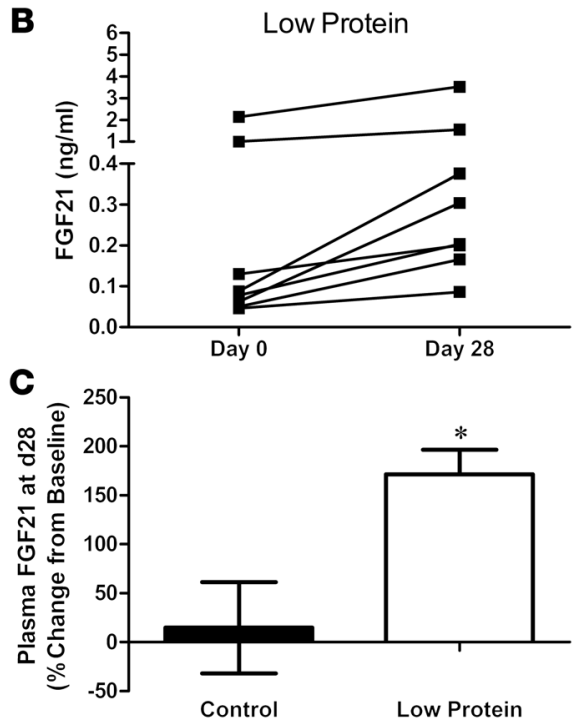

Figure 3. Dietary protein restriction increases FGF21 in humans. Plasma FGF21 concentrations were measured in human subjects given isocaloric amounts of diets that were either normal (A) or low (B) in protein for 28 days, and the change in FGF21 between baseline (day 0 ) and day 28 was compared (C). $n=8-9 /$ group; ${ }^{*} P=0.01$.

in hepatic Ppara mRNA expression during protein restriction in either rats or mice (Figure 4A), nor did we detect changes in the expression of genes downstream of PPAR $\alpha$ signaling (Figure 4B). In contrast, we detected a robust increase in the phosphorylation of eIF2 $\alpha$ within the liver following protein restriction $(P<0.01$; Figure $4 \mathrm{C}$ ), with eIF $2 \alpha$ phosphorylation being increased in each setting in which hepatic FGF21 expression was increased by protein restriction (Figure 4D). The serine/threonine kinase general control nonderepressible 2 (GCN2) phosphorylates eIF $2 \alpha$ in response to amino acid depletion, leading to the inhibition of general protein translation, but the activation of the amino acid response pathway (36-38). To test the role of PPAR $\alpha$ and GCN2 in LP-induced increases in FGF21, we compared circulating FGF21 concentrations in WT, Gcn2-KO and Ppara-KO mice after 4 days of LP diet. Deletion of $G c n 2$ or Ppara independently induced a marked reduction of circulating FGF21 on the control diet and greatly blunted LP-induced increases in FGF21 $(P<0.01$; Figure 4 E). Deletion of $G c n 2$ also reduced basal and LP-induced eIF $2 \alpha$ phosphorylation $(P<0.05$; Figure $4 \mathrm{~F})$, although LP still marginally increased eIF $2 \alpha$ phosphorylation in the absence of GCN2. LP-induced increases in eIF2 $\alpha$ phosphorylation were intact in Ppara-KO mice. These data collectively indicate that both PPAR $\alpha$ and GCN2 contribute to the regulation of circulating FGF21 during protein restriction.
FGF21 is required for the behavioral and metabolic response to protein restriction. To determine whether FGF21 is required for adaptive responses to protein restriction, WT and Fgf21-deficient mice were placed on a LP diet for 14 days. As expected (4, 31, 39-42), WT mice displayed both increased food intake (Figure 5E) and a progressive increase in EE (Figure $5 \mathrm{~A}$ ) following the switch to LP diet. Specifically, EE increased, by approximately $15 \%$ in WT mice by days 5 to $7(P<0.01$; Figure $5, C$ and $D)$, an effect detected during both the light and dark periods. The increase in EE was observed irrespective of whether the EE data were expressed on a per-animal basis (Figure 5C) or were normalized to body weight or lean mass (Supplemental Figure 4, A and B). In contrast, the LP diet had no effect on food intake or EE in Fgf21-KO mice (Figure 5, B-E). Analysis of the EE data using analysis of covariance (ANCOVA) with body weight as the covariant demonstrated a LP-dependent increase in EE in WT but not Fgf21-KO mice (Supplemental Figure 4, C and F). The respiratory exchange ratio (RER) was significantly increased (signifying an increase in the rate of carbohydrate oxidation) by the LP diet in both Fgf21-KO and WT mice ( $P=0.01$; Supplemental Figure 4D). Finally, locomotor activity (beam breaks) trended lower in Fgf21-KO mice relative to WT ( $P=0.09$; Supplemental Figure $4 \mathrm{E})$, but there were no effects of diet in either genotype.

The marked changes in feeding behavior and EE in Fgf21-KO mice resulted in clear differences in body weight and composition. WT mice fed the LP diet for 14 days exhibited a significant loss of body weight $(P=0.0024$; Figure $5 \mathrm{~F})$, explained by a decrease of fat mass gain and a loss of lean mass $(P=0.001$; Figure $5, G$ and $\mathrm{H})$. In contrast, Fgf21-KO mice maintained their weight during LP diet feeding relative to controls. Fgf21-KO mice also increased body fat gain on the diet $(P=0.06)$, and although their lean mass decreased slightly, the effect was smaller than was observed in WT controls. Collectively, these data suggest that FGF21 is required for adaptive changes in energy metabolism and growth during dietary protein restriction.

\section{Discussion}

FGF21 has received considerable attention due to its potent antidiabetic effects in rodent models, yet little is known regarding its physiological role. Previous work has implicated FGF21 in the metabolic response to starvation and a KD in rodent models $(6,7$, 34). Here, we provide evidence that the induction of FGF21 during starvation and KD is mediated primarily by reduced protein intake, with protein restriction producing a robust increase in circulating FGF21 in both rodents and humans. This rise in circulating FGF21 appears to derive largely from the liver and requires both PPAR $\alpha$ and GCN2/eIF2 $\alpha$. Furthermore, FGF21 is required for the effects of protein restriction on food intake, EE, and body weight. Taken together, these data demonstrate that FGF21 is a key mediator of the physiological response to dietary protein restriction (Figure 6).

Our data do not contradict prior work assessing the effects of starvation or KD on FGF21, but instead provide convincing evidence that reduced dietary protein provides a more parsimonious explanation for the observed increases in circulating FGF21. This redefinition of the dietary regulation of FGF21 also provides a mechanism to explain previous discrepancies regarding the regulation of FGF21. For example, despite the evidence in rodents that FGF21 is induced by KDs and starvation, similar work in humans 
A
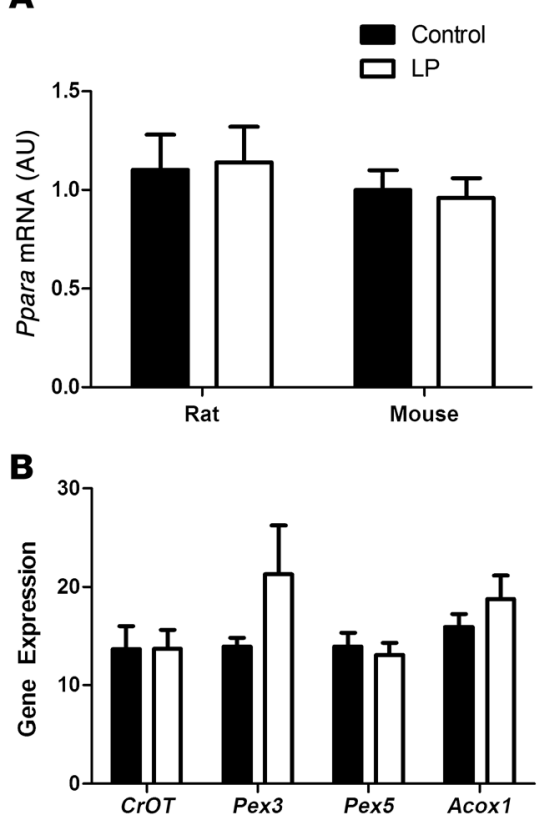

C

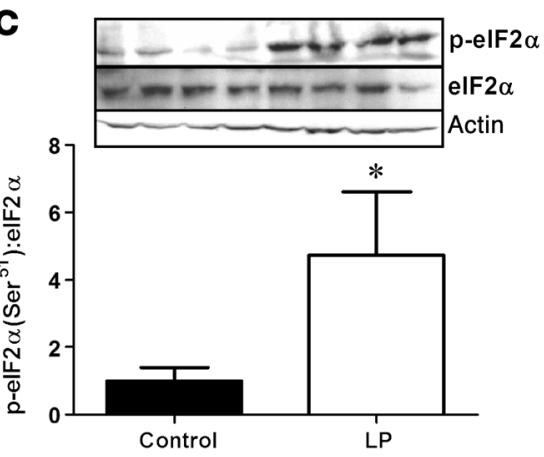

D

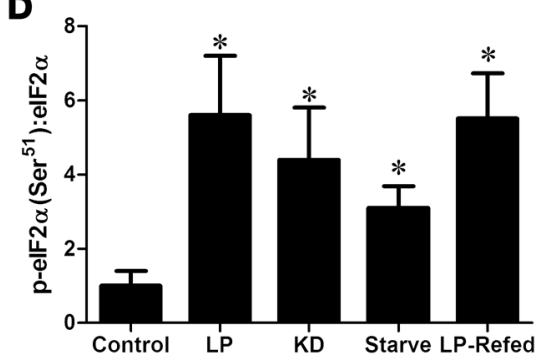

E

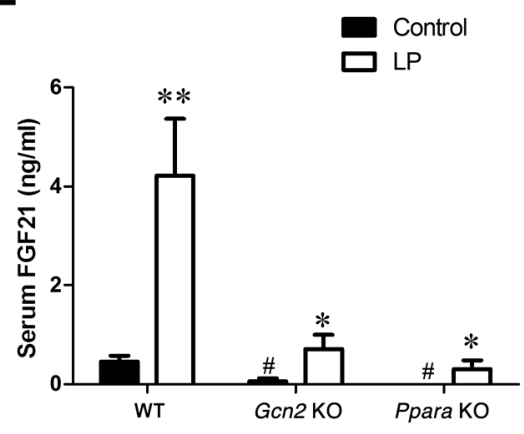

F

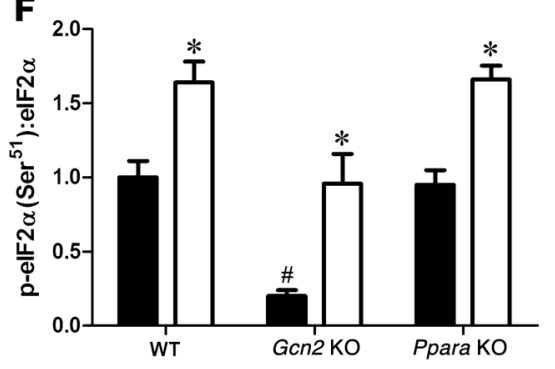

Figure 4. LP-induced increases in circulating FGF21 depend on both GCN2 and PPAR $\alpha$ signaling. Liver Ppara mRNA expression was assessed in mice and rats on LP diet for 14 days (A) as well as liver expression of PPAR $\alpha$ responsive genes in rats (B). Western blot of elF2 $\alpha$ phosphorylation in liver of rats consuming control or LP diet for 2 days (C), and elF2 $\alpha$ phosphorylation in liver of mice and rats during various models of protein restriction described previously (D). Serum FGF21 (E) and liver elF2 $\alpha$ phosphorylation (F) was measured in WT, Gcn2-KO, and Ppara-KO mice following 4 days of LP diet. $n=5-8 /$ group. ${ }^{*} P<0.05 ;{ }^{* *} P<0.01$ vs. control; ${ }^{*} P<0.05$ vs. WT control.

has been much less convincing. In particular, low-carbohydrate KDs have been without effect on FGF21 in humans, while short periods of fasting have produced mixed and relatively weak results $(21,23,25,26)$. In these human studies, protein intake is often more strongly controlled, and in at least one study, the subjects on the low-carbohydrate diet consumed similar absolute amounts of protein and actually increased consumption of protein relative to energy (25). Based on our data, we would not expect the manipulation of carbohydrate alone to affect FGF21. The observation that serum FGF21 increases on both the LP diet and KD also provides insight into the role of individual macronutrients in regulating FGF21. The LP diet is high in carbohydrate, but equal in fat content relative to the control diet, while the KD diet is virtually devoid of carbohydrate and very high in fat. Thus, FGF21 is increased in settings of both high and low carbohydrate and high and low fat, with only the reduction of protein being common between these dietary conditions.

Considering the opposing effects of protein versus energy restriction on hepatic FGF21 expression, our observations not only suggest that protein restriction increases hepatic expression of FGF21, but also that energy restriction may actually decrease FGF21 expression as long as protein deficiency is prevented. Stated differently, our data support the hypothesis that protein and energy may independently and antagonistically regulate hepatic FGF21 gene expression. The underlying reason for this differential regulation remains unclear, but it seems likely that hepatic metabolism is uniquely different in settings of protein versus energy restriction. For instance, our data are consistent with previous work (33) demonstrating that protein restriction triggers adaptive increases in hepatic amino acid synthesis and decreases in hepatic amino acid catabolism, whereas these changes do not occur during energy restriction. Based on this evidence for unique differences in hepatic metabolism between protein and energy restriction, our data are consistent with the hypothesis that reductions in dietary amino acid supply to the liver activate cellular pathways (such as GCN2/eIF2 $\alpha$ ) that are distinct from those pathways activated by energy restriction, with these unique pathways contributing to both the induction of hepatic FGF21 and the physiological response to dietary protein restriction.

Prior work implicates the transcription factor PPAR $\alpha$ in the regulation of FGF21 $(7,8,25,26)$, and we found that deletion of Ppara produced a marked reduction in serum FGF21 on both control and LP diets. However, we found little evidence suggesting that protein restriction, in the absence of energy restriction, increases PPAR $\alpha$ activity. While PPAR $\alpha$ may play a constitutive role in maintaining normal levels of hepatic FGF21 expression, it is not clear whether it is involved in the response to a LP diet. By comparison, GCN2-dependent phosphorylation of eIF2 $\alpha$ is a key signaling response linking amino acid restriction to the regulation of hepatic metabolism (36-38, 43-48). Recent studies indicate that the FGF21 promoter contains 2 amino acid response elements (AARE) that are bound by the transcription factor ATF 4 in response to increases of eIF2 $\alpha$ phosphorylation during ER stress or amino acid restriction (27-30). Thus, eIF2 $\alpha$-dependent activation of ATF 4 provides a potential molecular link between amino acid restriction and hepatic FGF21 induction in vivo. Our observations that eIF2 $\alpha$ phosphorylation is increased in multiple settings of dietary protein restriction and that hepatic Fgf 21 mRNA levels are reduced in GCN2-deficient mice implicate GCN2 and eIF $2 \alpha$ as mediators of the effect of protein restriction. 

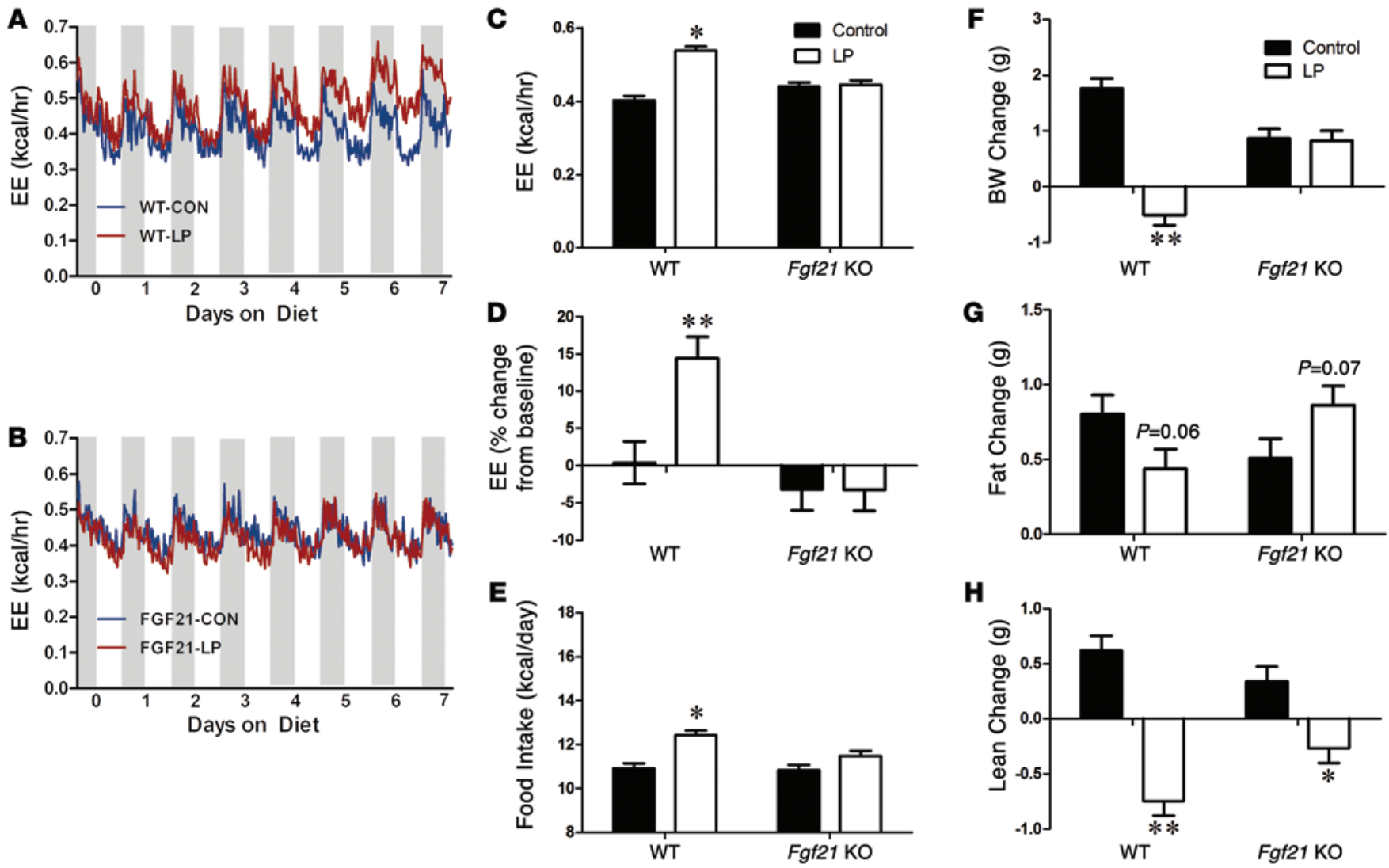

Figure 5. FGF21 is required for LP-induced changes in EE, food intake, body weight, and body composition. EE in WT (A) and Fgf21-KO (B) mice consuming control or LP diet. Average EE (C) and change in EE from baseline (D) over days 5 to 7 of diet consumption. Average daily food intake (E) in WT and Fgf21-KO mice over the 14 days of diet exposure. Change in body weight (F), fat mass (G), and lean mass (H) in WT and Fgf21-KO mice on control or LP diet for 14 days. $n=10 /$ group, ${ }^{*} P<0.05$; ${ }^{* *} P<0.01$.

It should be noted, however, that while serum FGF21 concentrations are reduced in both Ppara-KO and Gcn2-KO mice, LP diet nevertheless increases circulating FGF21 even in the absence of either PPAR $\alpha$ or GCN2. Similarly, although deletion of GCN2 reduces hepatic eIF $2 \alpha$ phosphorylation, the LP diet still induces a relative increase of hepatic eIF $2 \alpha$ phosphorylation in the absence of GCN2. When taken together, these data suggest that additional signaling systems beyond PPAR $\alpha$ and GCN2 likely converge on both eIF2 $\alpha$ phosphorylation and FGF21 expression in response to protein restriction, and further work is required to identify all of the pathways involved. Finally, while phosphorylation of eIF2 $\alpha$ is a primary cellular mechanism for the inhibition of protein synthesis, it seems likely that some residual protein synthesis remains in animals consuming the LP diet, considering that the LP diet increased both hepatic eIF2 $\alpha$ phosphorylation and serum FGF21 protein levels.

Previous experiments by our group and others demonstrate that protein restriction increases food intake in mice, rats, and humans $(3,4,31,40)$, and WT mice in this study also increased food intake on the LP diet. The LP diet also increased EE, a previously established response to protein restriction that presumably involves activation of the sympathetic nervous system $(41,42)$. The restriction of protein intake coupled with elevated EE resulted in a marked reduction of weight gain in WT mice, including reductions in both lean and fat mass relative to control mice. Impressively, neither food intake nor EE was altered by LP in Fgf21-KO mice. More remarkably, Fgf21-KO mice also exhibited no change in body weight, and actually increased fat mass on the LP diet. These data suggest that mice cannot detect and/or respond to dietary protein restriction in the absence of FGF21. Prior experiments have linked FGF21 to overall body growth by demonstrating reduced body size in transgenic mice overexpressing FGF21 (49). The effect appears to be mediated in part by FGF21dependent inhibition of growth hormone $(\mathrm{GH})$ signaling in the liver, resulting in reduced hepatic IGF1 secretion (49-51). A separate study indicates that FGF21 contributes to the attenuation of growth during undernutrition (52). Our results support and extend these data and demonstrate that FGF21 plays an essential role in linking dietary protein intake to body growth and composition.

The mechanism underlying the FGF21-dependent increases in food intake and EE during protein restriction is currently unclear, but it seems likely that at least a portion of these effects are mediated by a direct action of FGF21 on the brain. Indeed, previous experiments have demonstrated that infusion of FGF21 directly into the brain is sufficient to increase both food intake and EE (18), and more recent work indicates that FGF21 action in the brain is an important mechanism of its effects on circadian activity, insulin sensitivity, reproduction, and growth $(19,53)$. However, it also possible that FGF21 acts directly on brown adipose tissue (BAT) or WAT to induce thermogenesis (13). Additional experiments are required to define the specific sites and mechanisms through which FGF21 increases EE in animals on a LP diet. 


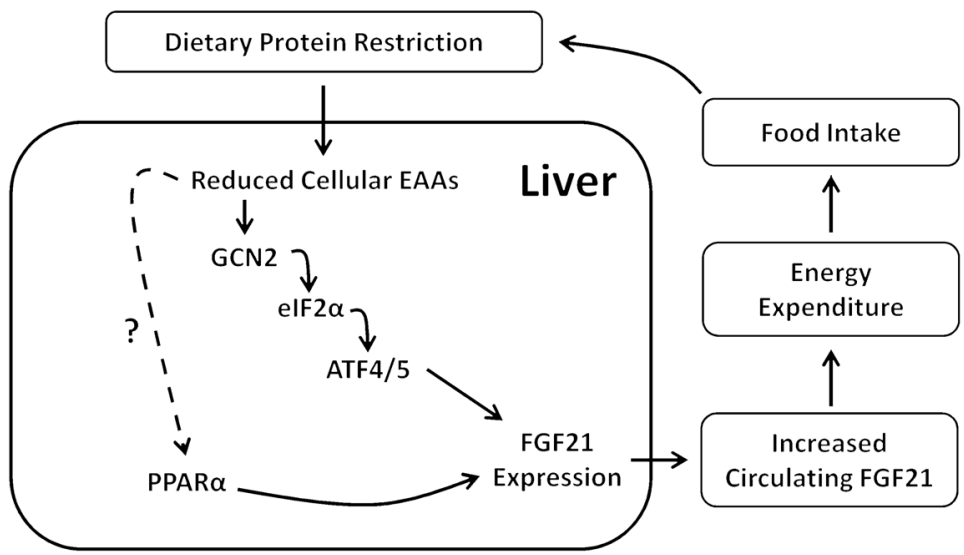

Figure 6. Hypothetical model of protein-dependent regulation of FGF21 and its effects on food intake and EE. Reduced consumption of dietary protein leads to reduced delivery of amino acids to the liver, activating the kinase GCN2 and leading to increased elF2 $\alpha$ phosphorylation and activation of ATF4/ATF5. ATF4/5 bind AAREs within the FGF21 promoter, leading to increased liver FGF21 production and increased circulating FGF21. PPAR $\alpha$ is also required for normal levels of circulating FGF21. Finally, increased circulating levels of FGF21 increase EE and food intake and also alter body weight gain and body composition.
Considering recent genome-wide association studies linking FGF21 to macronutrient selection in humans $(54,55)$, our data strongly suggest that FGF21 plays an important role in the detection or response to protein restriction and macronutrient imbalance in rodents and humans. The ability to couple metabolism, food intake, and body growth to variations in nutrient intake is key to survival in periods of nutrient deficiency. Although the mechanisms underlying adaptive responses to protein restriction are poorly defined (4), recent studies highlight the impact of dietary protein intake on health, metabolism, and life span $(56,57)$. Our data not only demonstrate that variations in dietary protein content exert important effects on metabolism and behavior; they also define a role for FGF21 in this adaptive response.

\section{Methods}

Animals and diets. Male Sprague Dawley rats (Harlan) and male C57BL/6 mice (Jackson Lab) were used in all studies. Gcn2- and Ppara-deficient mice on the C57BL/6 background were purchased from Jackson Laboratory. Fgf21-deficient mice on the C57BL/6 background were provided by Steven Kliewer (University of Texas Southwestern, Dallas, Texas, USA) (6). Control and LP diets were formulated and produced by Research Diets and were designed to be isocaloric by equally varying protein and carbohydrate content while keeping fat constant. Control diet contained $20 \%$ casein (by weight) as the protein source, while in rats, the LP diet contained $10 \%$ casein and, in mice, the LP diet contained $5 \%$ casein. In our experience, mice require a lower percentage of protein to produce consistent increases in EE. On an energy basis, the diets contained protein at $18 \%$ (control), $9 \%$ (rat LP), and $4 \%$ (mouse LP). A detailed dietary composition is provided in Supplemental Table 1. The KD was purchased from Bio-Serv (F3666) to replicate previous work (7, 34 ), and on an energy basis, the KD contained $4.7 \%$ protein, $93.4 \%$ fat and $1.8 \%$ carbohydrate. Animals were single housed in 12-hour light/12-hour dark cycle with ad libitum access to food or water unless otherwise noted.

For general effects of dietary protein restriction, single-housed rats or mice were transferred from chow to the control diet for approximately 5 days, at which point a random subgroup of animals were transferred to LP diet. Animals consumed control or LP diet ad libitum for the duration of the study, at which time animals were sacrificed and liver, eWAT, and skeletal muscle collected and snap-frozen for further analysis. Mice and rats were sacrificed during the mid-light cycle in the fed state using acute exposure to $\mathrm{CO}_{2}$ followed by rapid decapitation. Trunk blood was also collected at sacrifice, allowed to clot overnight at $4^{\circ} \mathrm{C}$, and centrifuged at $3000 \mathrm{~g}$; serum was then collected.

Comparison of energy versus protein restriction. Rats were adapted to the control diet and then placed on one of 4 diet regimens to independently control protein versus energy intake: (a) NP:NE, ad libitum consumption of the control diet (20\% casein); (b) LP:NE, Ad libitum consumption of our standard LP diet (10\% casein), which represents a $50 \%$ restriction of dietary protein; (c) NP:LE, a $40 \%$ casein diet offered at $50 \%$ of the ad libitum intake of the control group, producing protein intake equal to control, but a $50 \%$ restriction of energy intake; or (d) LP:LE, control diet limited to $50 \%$ of ad libitum levels, thus representing $50 \%$ restriction of protein and energy. Because all diets were isocaloric and equal in energy density, restriction of energy intake in the NP:LE and LP:LE groups was accomplished by pair-feeding food intake to $50 \%$ of the NP:NE group each day. Rats consumed the diets for 4 days and were then sacrificed and tissue collected as described above. Energy intake, protein intake, and body weights for these rats are presented in Supplemental Figure 2, A-C.

Effects of food deprivation and refeeding. Rats were adapted to the control diet and then placed on one of 4 diet regimens: (a) ad libitum intake (fed); (b) 48-hour food deprivation (starved); (c) 48-hour food deprivation followed by 4 hours of refeeding on a $5 \%$ casein diet (LP refed); or (d) 48-hour food deprivation followed by 4 hours of refeeding on the $40 \%$ casein diet (HP refed). The 2 refed groups were offered a fixed amount of food (12 g) during the 4 hours of refeeding and thus consumed an equal volume and energy. Refeeding began at lights off, and ad libitum-fed rats also consumed a statistically similar, although slightly smaller, amount of food during this 4 -hour period prior to sacrifice. After 4 hours, rats were sacrificed and tissue collected as described above. Energy and protein intake over the 4 hours of refeeding and final body weights for these rats are presented in Supplemental Figure 3.

Effect of KD supplemented with carbohydrate or protein. Mice were adapted to the control diet and then placed on one of 4 dietary regimens: (a) control diet; (b) KD alone; (c) KD supplemented with sucrose $(\mathrm{KD}+\mathrm{CHO})$; or (d) KD supplemented with casein $(\mathrm{KD}+\mathrm{P})$. The sucrose and casein were manually mixed into the KD to produce a homogenous consistency and were added at a ratio of $1 \mathrm{~g}$ sucrose/ casein per $10 \mathrm{~g} \mathrm{KD}$. All diets were offered ad libitum in food jars.

Role of PPAR $\alpha$ and GCN2 in LP-induced increases in FGF21. PPAR $\alpha$ deficient mice (Ppara-KO) and GCN2-deficient mice (Gcn2-KO) were adapted to the control diet and placed on control or LP diet for 4 days. 
On day 4 , mice were sacrificed and tissue was collected as described above ( $n=6-8$ per group). At sacrifice, body weights in both $G c n 2$ mice (control: $23.06 \pm 0.52 \mathrm{~g}, \mathrm{LP}: 22.18 \pm 0.49 \mathrm{~g}, P=0.238$ ) and Ppara (control: $21.1 \pm 0.61 \mathrm{~g}$, LP: $21.9 \pm 0.83 \mathrm{~g}, P=0.465)$ were similar between diet groups. Food intake over the 4 days was also not significantly different (GCN2, control: $2.7 \pm 0.08 \mathrm{~g}, \mathrm{LP}: 2.6 \pm 0.04 \mathrm{~g}, P=0.432$; PPAR $\alpha$, control: $2.3 \pm 0.07 \mathrm{~g}$, LP: $2.6 \pm 0.08 \mathrm{~g}, P=0.124)$. Body weights and food intakes were in the normal range and similar to those of WT controls, indicating that neither Gcn2 nor Ppara mice exhibited gross changes in growth rate or food intake.

Role of FGF 21 in response to LP diet. FGF21-deficient ( Fgf $21-\mathrm{KO})$ and WT controls were adapted to control diet and then randomly placed on either control or LP for 14 days (10 mice/diet/genotype). Mice were initially adapted to the control diet while in metabolic chambers (PhenoMaster/LabMaster; TSE Systems), were transitioned to the LP diet while in the metabolic chambers, and remained there for the first 7 days of dietary exposure. Afterward, mice were returned to standard caging for the final 7 days and, after 14 days on diet,were sacrificed and tissue collected as described above. Body weight and food intake were collected every 2 days through the 14-day experiment. Body composition was measured via TD-NMR (Bruker Minispec) on the start of experimental diets (day 0), the final day of metabolic analysis (day 7), and on the day of sacrifice (day 14).

FGF21 analysis in protein-restricted humans. Banked human plasma samples were utilized from a randomized controlled clinical trial (clinicaltrials.gov identifier NCT00565149). Details of the experimental design and primary outcomes have been previously published (35). Briefly, male and female inpatient subjects were placed on a weightstabilization diet, and then randomized to 3 diets containing 5\% (LP), $15 \%$ (control), or $25 \%$ protein for 56 days. During this time, subjects were overfed, resulting in a $40 \%$ increase in energy intake in all groups. Despite the overfeeding, the LP group remained protein restricted by roughly $50 \%$ compared with baseline. FGF21 protein concentrations in plasma samples collected following an overnight fast were measured in the $5 \%$ (LP) and $15 \%$ (control) groups at baseline and after 28 days on diet. FGF21 levels were measured via a human-specific ELISA (BioVendor, see below). Change in FGF21 was analyzed by comparing day 0 versus day 28 values within a diet group (paired, 2-tailed $t$ test), and by comparing percentage change from baseline (day 0) between groups. For the final analysis, 3 subjects in the control group and 2 subjects in the LP group exhibited day 0 and day 28 FGF21 values that were well above those of the other subjects. Their values fell above the range of the standard curve, necessitating that their plasma samples be reassayed following serial dilution to obtain accurate measures. The observation of a high degree of variability in circulating FGF21 concentrations is consistent with previous work in humans (26).

Western immunoblot analysis. Frozen liver blocks were homogenized and sonicated for protein extraction in $300 \mu \mathrm{l}$ lysis buffer containing 50 $\mathrm{mM} \mathrm{KCl}$ (Acros Organics), $25 \mathrm{mM}$ HEPES (Acros Organics), $125 \mu \mathrm{M}$ DTT (Sigma-Aldrich), $1 \mathrm{mM}$ PMSF (Thermo Scientific), $1 \mathrm{mM} \mathrm{Na}_{3} \mathrm{VO}_{4}$ (Acros Organics), 1\% (v/v) NP-40 Alternative (Merck), and protease inhibitor cocktail tablets (1 tablet/50 ml buffer; Roche) on ice. After centrifugation $\left(4^{\circ} \mathrm{C}, 10\right.$ minutes, $\left.13,000 \mathrm{~g}\right)$, the supernatant was collected and the protein content was quantified with Coomassie Plus (Bradford) Protein Assay Kit (Thermo Scientific) according to the manufacturer's protocol. For SDS-gel electrophoresis, $50 \mu \mathrm{g}$ sample solutions were diluted to $5 \times$ Laemmli sample buffer containing 60 mM Tris- $\mathrm{Cl}$ (pH 6.8), 2\% (w/v)
SDS, 10\% (v/v) glycerol, 5\% (v/v) 2-mercaptoethanol, and 0.01\% (v/v) bromophenol blue. The samples were heated at $95^{\circ} \mathrm{C}$ for 3 minutes and electrophoresed through a $10 \%$ precast polyacrylamide gel (Bio-Rad). Proteins were then transferred to $0.45-\mu \mathrm{m}$ pore size nitrocellulose membranes (Thermo Scientific). Membranes were blocked with 3\% (w/v) bovine serum albumin in TBST buffer $(20 \mathrm{mM}$ Tris/ $\mathrm{HCl}, 0.9 \%(\mathrm{w} / \mathrm{v})$ $\mathrm{NaCl}, 0.05 \%(\mathrm{v} / \mathrm{v})$ Tween-20 (pH 7.6) and incubated with the primary antibodies against eIF2 $\alpha$ (Abcam Inc; dilution: 1:1000), phospho-eIF2 $\alpha$ Ser $^{51}$ (EMD Millipore; dilution: 1:1000), or with $\alpha$-tubulin antibody (Abcam Inc; dilution: 1:10,000) at $4^{\circ} \mathrm{C}$ for 12 hours. Membranes were then washed with TBST, incubated with the corresponding HRP-conjugated anti-mouse (1:4000) or anti-rabbit IgG (1:2000) for 60 minutes at room temperature. After washing with TBST, the membranes were transferred to enhanced chemiluminescence substrate (Western Lightning-ECL; PerkinElmer Inc.) for 2 minutes and exposed to Premium X-Ray Film (F-BX57; Phenix Research Products) for 2 to 30 minutes. Bands were scanned and quantified using Image 1.47 (Wayne Rasband, National Institutes of Health). The level of phosphorylation of eIF $2 \alpha$ was calculated relative to the total amount of this enzyme.

Immunoassay determination of FGF21. Concentrations of FGF21 in serum were determined in mice and rats with an ELISA according to the procedure recommended by the manufacturer (no. RD291108200R, Mouse and Rat FGF-21 ELISA; BioVendor). The minimal detectable concentration of FGF21 with this assay was $18.4 \mathrm{pg} / \mathrm{ml}$. The intra- and interassay coefficients of variation for the mouse and rat FGF21 assay were less than $9.0 \%$ and less than $8.0 \%$, respectively. For determination of serum FGF21, $50 \mu \mathrm{l}$ of serum was diluted in $200 \mu \mathrm{l}$ of dilution buffer before analysis. Human FGF21 plasma concentrations were determined with an ELISA according to the procedure recommended by the manufacturer (no. RD191108200R, Human FGF21 ELISA;BioVendor). The minimal detectable concentration of FGF21 with this assay was 7.0 $\mathrm{pg} / \mathrm{ml}$. The intra- and interassay coefficients of variation for the human FGF21 assay were less than 4.0\%. For determination of plasma FGF21, $125 \mu \mathrm{l}$ of plasma was diluted in $125 \mu$ l of dilution buffer before analysis.

Analysis of BHBA. Mouse serum BHBA concentrations were determined by a colorimetric assay kit according to the procedure recommended by the manufacturer (no. 700190; Cayman Chemical Co.). The dynamic range of the kit is 0 to $0.5 \mathrm{mM} \mathrm{BHBA}$. The intra- and interassay coefficients of variation were $4.05 \%$ and $3.18 \%$, respectively. For determination of serum BHBA, $22 \mu \mathrm{l}$ of serum was diluted in $88 \mu \mathrm{l}$ of assay buffer before analysis.

Plasma amino acid measures. Plasma amino acids were measured using fluorometric HPLC via methods described previously $(58,59)$. Separation of the o-phthaldialdehyde amino acid derivatives was performed by gradient elution from a Supelcosil LC-18 column $(15 \mathrm{~cm} \times$ $4.6 \mathrm{~mm}, 3 \mu \mathrm{m}$; Sigma-Aldrich).

Real-time PCR. RNA extraction and real-time PCR were conducted as described previously (31). Total RNA was extracted from mediobasal hypothalamus using TRIzol reagent following the manufacturer's protocol (15596018; Invitrogen). RNA quality and quantity were determined by spectrophotometry using a NanoDrop (Thermo Scientific). cDNA synthesis was performed with M-MLV reverse transcriptase (M1701; Promega), and mRNA was quantified on the ABI 7900 platform using SYBR Green methodology in optical 384-well plates (Applied Biosystems). Primer pairs were designed using NCBI Primer-BLAST with at least 1 primer spanning an exon-exon boundary. Target gene expression was normalized with cyclophilin B as the endogenous control. 
Statistics. Data were analyzed using the SAS software package (SAS V9;SAS Institute) using 1- or 2-way ANOVA or repeated measures ANOVA, using the general linear model procedure. When experimentwide tests were significant, post-hoc comparisons were made using the LSMEANS statement with the PDIFF option. Analysis of EE with body weight as the covariate was assessed via ANCOVA using the general linear model procedure of SAS, with results validated against the MMPC.org ANCOVA data analysis tool. Human FGF21 was analyzed by comparing day 0 versus day 28 values within a diet group (paired, 2-tailed $t$ test) and comparing percentage change from baseline (day 0 ) among groups. All data are expressed as mean \pm SEM, with a probability value of 0.05 considered statistically significant.

Study approval. Procedures involving animals were approved by the PBRC Institutional Animal Care and Use Committee. Procedures involving human subjects were reviewed by an external advisory committee and approved by the PBRC Institutional Review Board and the US Department of Agriculture. Safety was monitored by Donna Ryan (associate director of clinical research, PBRC). Participants provided written informed consent.

\section{Acknowledgments}

The authors would like to thank Alex Navard, Anne-Victoria Traylor, Jessie W. Davidson, John Hedgepeth, Adele Addington, and the staff of the PBRC Comparative Biology Core for their skillful assistance and excellent technical support. C.D. Morrison was supported by R01DK081563. T. Laeger was supported by a research fellowship from the Deutsche Forschungsgemeinschaft (DFG), LA 3042/2-1. This project/work used facilities within the Animal Metabolism and Behavior Core, Genomics Core, and Cell Biology and Bioimaging Core at PBRC that are supported in part by COBRE (P2OGM103528) and NORC (P3ODK072476) center grants from the NIH. The authors utilized the MMPC.org data analysis tool (http://www.mmpc.org/shared/regression.aspx) for assistance with ANCOVA analysis of EE.

Address correspondence to: Christopher D. Morrison, Associate Professor, Pennington Biomedical Research Center, 6400 Perkins Road, Baton Rouge, Louisiana 70808, USA. Phone: 225.763.3145; E-mail:morriscd@pbrc.edu.
1. Simpson SJ, Raubenheimer D. Geometric analysis of macronutrient selection in the rat. Appetite. 1997;28(3):201-213.

2. Sorensen A, Mayntz D, Raubenheimer D, Simpson SJ. Protein-leverage in mice: the geometry of macronutrient balancing and consequences for fat deposition. Obesity (Silver Spring). 2008;16(3):566-571.

3. Gosby AK, et al. Testing protein leverage in lean humans: a randomised controlled experimental study. PLoS One. 2011;6(10):e25929.

4. Morrison CD, Reed SD, Henagan TM. Homeostatic regulation of protein intake: in search of a mechanism. Am J Physiol Regul Integr Comp Physiol. 2012;302(8):R917-R928.

5. Potthoff MJ, Kliewer SA, Mangelsdorf DJ. Endocrine fibroblast growth factors $15 / 19$ and 21 : from feast to famine. Genes Dev. 2012;26(4):312-324.

6. Potthoff MJ, et al. FGF21 induces PGC-1 $\alpha$ and regulates carbohydrate and fatty acid metabolism during the adaptive starvation response. Proc Natl Acad Sci U S A. 2009;106(26):10853-10858.

7. Badman MK, Pissios P, Kennedy AR, Koukos G, Flier JS, Maratos-Flier E. Hepatic fibroblast growth factor 21 is regulated by $\operatorname{PPAR} \alpha$ and is a key mediator of hepatic lipid metabolism in ketotic states. Cell Metab. 2007;5(6):426-437.

8. Inagaki $\mathrm{T}$, et al. Endocrine regulation of the fasting response by PPAR $\alpha$-mediated induction of fibroblast growth factor 21. Cell Metab. 2007;5(6):415-425.

9. Kharitonenkov A, et al. The metabolic state of diabetic monkeys is regulated by fibroblast growth factor-21. Endocrinology. 2007;148(2):774-781.

10. Kharitonenkov A, et al. FGF-21 as a novel metabolic regulator. J Clin Invest. 2005;115(6):1627-1635.

11. Coskun T, et al. Fibroblast growth factor 21 corrects obesity in mice. Endocrinology. 2008;149(12):6018-6027.

12. Xu J, et al. Fibroblast growth factor 21 reverses hepatic steatosis, increases energy expenditure, and improves insulin sensitivity in diet-induced obese mice. Diabetes. 2009;58(1):250-259.

13. Fisher FM, et al. FGF21 regulates PGC- $1 \alpha$ and browning of white adipose tissues in adaptive thermogenesis. Genes Dev. 2012;26(3):271-281.

14. Zhang Y, et al. The starvation hormone, fibroblast growth factor-21, extends lifespan in mice. Elife. 2012;1:e00065.

15. Fisher FM, et al. Integrated regulation of hepatic metabolism by fibroblast growth factor 21 (FGF21) in vivo. Endocrinology. 2011;152(8):2996-3004.

16. Camporez JP, et al. Cellular mechanisms by which FGF21 improves insulin sensitivity in male mice. Endocrinology. 2013;154(9):3099-3109.

17. Hotta Y, et al. Fibroblast growth factor 21 regulates lipolysis in white adipose tissue but is not required for ketogenesis and triglyceride clearance in liver. Endocrinology. 2009;150(10):4625-4633.

18. Sarruf DA, et al. Fibroblast growth factor 21 action in the brain increases energy expenditure and insulin sensitivity in obese rats. Diabetes. 2010;59(7):1817-1824.

19. Bookout AL, et al. FGF21 regulates metabolism and circadian behavior by acting on the nervous system. Nat Med. 2013;19(9):1147-1152.

20. Gaich G, et al. The effects of LY2405319, an FGF21 analog, in obese human subjects with type 2 diabetes. Cell Metab. 2013;18(3):333-340.

21. Angelin B, Larsson TE, Rudling M. Circulating fibroblast growth factors as metabolic regulators-a critical appraisal. Cell Metab. 2012;16(6):693-705.

22. Chavez AO, Molina-Carrion M, Abdul-Ghani MA, Folli F, Defronzo RA, Tripathy D. Circulating fibroblast growth factor- 21 is elevated in impaired glucose tolerance and type 2 diabetes and correlates with muscle and hepatic insulin resistance. Diabetes Care. 2009;32(8):1542-1546.

23. Dushay J, et al. Increased fibroblast growth factor 21 in obesity and nonalcoholic fatty liver disease. Gastroenterology. 2010;139(2):456-463.

24. Zhang X, et al. Serum FGF21 levels are increased in obesity and are independently associated with the metabolic syndrome in humans. Diabetes.
2008;57(5):1246-1253.

25. Christodoulides C, Dyson P, Sprecher D, Tsintzas $\mathrm{K}$, Karpe F. Circulating fibroblast growth factor 21 is induced by peroxisome proliferator-activated receptor agonists but not ketosis in man. J Clin Endocrinol Metab. 2009;94(9):3594-3601.

26. Galman C, et al. The circulating metabolic regulator FGF21 is induced by prolonged fasting and PPAR $\alpha$ activation in man. Cell Metab. 2008;8(2):169-174.

27. Schaap FG, Kremer AE, Lamers WH, Jansen PL, Gaemers IC. Fibroblast growth factor 21 is induced by endoplasmic reticulum stress. Biochimie. 2013;95(4):692-699.

28. Kim KH, et al. Metformin-induced inhibition of the mitochondrial respiratory chain increases FGF21 expression via ATF4 activation. Biochem Biophys Res Commun. 2013;440(1):76-81.

29. Keipert S, et al. Skeletal muscle mitochondrial uncoupling drives endocrine cross-talk through the induction of FGF21 as a myokine. Am JPhysiol Endocrinol Metab. 2014;306(5):E469-E482.

30. De Sousa-Coelho AL, Marrero PF, Haro D. Activating transcription factor 4 -dependent induction of FGF21 during amino acid deprivation. Biochem J. 2012;443(1):165-171.

31. Morrison CD, Xi X, White CL, Ye J, Martin RJ. Amino acids inhibit Agrp gene expression via an mTOR-dependent mechanism. Am J Physiol Endocrinol Metab. 2007;293(1):E165-E171.

32. White BD, Porter MH, Martin RJ. Effects of age on the feeding response to moderately low dietary protein in rats. Physiol Behav. 2000;68(5):673-681.

33. Kalhan SC, et al. Metabolic and genomic response to dietary isocaloric protein restriction in the rat. J Biol Chem. 2011;286(7):5266-5277.

34. Badman MK, Koester A, Flier JS, Kharitonenkov A, Maratos-Flier E. Fibroblast growth factor 21-deficient mice demonstrate impaired adaptation to ketosis. Endocrinology. 2009;150(11):4931-4940.

35. Bray GA, et al. Effect of dietary protein content 
on weight gain, energy expenditure, and body composition during overeating: a randomized controlled trial. JAMA. 2012;307(1):47-55.

36. Kilberg MS, Balasubramanian M, Fu L, Shan J. The transcription factor network associated with the amino acid response in mammalian cells. Adv Nutr. 2012;3(3):295-306.

37. Chaveroux C, et al. Molecular mechanisms involved in the adaptation to amino acid limitation in mammals. Biochimie. 2010;92(7):736-745.

38. Wek RC, Jiang HY, Anthony TG. Coping with stress: eIF2 kinases and translational control. Biochem Soc Trans. 2006;34(pt 1):7-11.

39. White BD, He B, Dean RG, Martin RJ. Low protein diets increase neuropeptide $Y$ gene expression in the basomedial hypothalamus of rats. J Nutr. 1994;124(8):1152-1160.

40. White BD, Porter MH, Martin RJ. Protein selection, food intake, and body composition in response to the amount of dietary protein. Physiol Behav. 2000;69(4):383-389.

41. Rothwell NJ, Stock MJ. Influence of carbohydrate and fat intake on diet-induced thermogenesis and brown fat activity in rats fed low protein diets. J Nutr. 1987;117(10):1721-1726.

42. Rothwell NJ, Stock MJ, Tyzbir RS. Mechanisms of thermogenesis induced by low protein diets. Metabolism. 1983;32(3):257-261.

43. Anthony TG, et al. Preservation of liver protein synthesis during dietary leucine deprivation occurs at the expense of skeletal muscle mass in mice deleted for eIF2 kinase GCN2.J Biol Chem. 2004;279(35):36553-36561.

44. Maurin AC, et al. The GCN2 kinase biases feeding behavior to maintain amino acid homeostasis in omnivores. Cell Metab. 2005;1(4):273-277.

45. Zhang P, et al. The GCN2 eIF2 $\alpha$ kinase is required for adaptation to amino acid deprivation in mice. Mol Cell Biol. 2002;22(19):6681-6688.

46. Guo F, Cavener DR. The GCN2 eIF2 $\alpha$ kinase regulates fatty-acid homeostasis in the liver during deprivation of an essential amino acid. Cell Metab. 2007;5(2):103-114.

47. Dudek SM, Semenkovich CF. Essential amino acids regulate fatty acid synthase expression through an uncharged transfer RNA-dependent mechanism. JBiol Chem.1995;270(49):29323-29329.

48. Xiao F, et al. Leucine deprivation increases hepatic insulin sensitivity via GCN2/mTOR/S6K1 and AMPK pathways. Diabetes. 2011;60(3):746-756.

49. Inagaki T, Lin VY, Goetz R, Mohammadi M, Mangelsdorf DJ, Kliewer SA. Inhibition of growth hormone signaling by the fasting-induced hormone FGF21. Cell Metab. 2008;8(1):77-83.

50. Yu J, et al. Growth hormone stimulates transcription of the fibroblast growth factor 21 gene in the liver through the signal transducer and activator of transcription 5. Endocrinology. 2012;153(2):750-758.

51. Chen W, et al. Growth hormone induces hepatic production of fibroblast growth factor 21 through a mechanism dependent on lipolysis in adipo- cytes. J Biol Chem. 2011;286(40):34559-34566.

52. Kubicky RA, Wu S, Kharitonenkov A, De Luca F. Role of fibroblast growth factor 21 (FGF21) in undernutrition-related attenuation of growth in mice. Endocrinology. 2012;153(5):2287-2295.

53. Owen BM, et al. FGF21 contributes to neuroendocrine control of female reproduction. Nat Med. 2013;19(9):1153-1156.

54. Tanaka T, et al. Genome-wide meta-analysis of observational studies shows common genetic variants associated with macronutrient intake. Am JClin Nutr. 2013;97(6):1395-1402.

55. Chu AY, et al. Novel locus including FGF21 is associated with dietary macronutrient intake. Hum Mol Genet. 2013;22(9):1895-1902.

56. Levine ME, et al. Low protein intake is associated with a major reduction in IGF-1, cancer, and overall mortality in the 65 and younger but not older population. Cell Metab. 2014;19(3):407-417.

57. Solon-Biet SM, et al. The ratio of macronutrients, not caloric intake, dictates cardiometabolic health, aging, and longevity in ad libitum-fed mice. Cell Metab. 2014;19(3):418-430.

58. Wu G, Knabe DA. Free and protein-bound amino acids in sow's colostrum and milk. J Nutr. 1994;124(3):415-424.

59. Purpera MN, et al. Impaired branched chain amino acid metabolism alters feeding behavior and increases orexigenic neuropeptide expression in the hypothalamus. JEndocrinol. 2012;212(1):85-94. 